\title{
Impediments to mixing classical and quantum dynamics
}

\author{
J. Caro and L.L. Salcedo \\ Departamento de Física Moderna \\ Universidad de Granada \\ E-18071 Granada, Spain
}

(August 7, 2018)

\begin{abstract}
The dynamics of systems composed of a classical sector plus a quantum sector is studied. We show that, even in the simplest cases, (i) the existence of a consistent canonical description for such mixed systems is incompatible with very basic requirements related to the time evolution of the two sectors when they are decoupled. (ii) The classical sector cannot inherit quantum fluctuations from the quantum sector. And, (iii) a coupling among the two sectors is incompatible with the requirement of physical positivity of the theory, i.e., there would be positive observables with a non positive expectation value.
\end{abstract}

PACS numbers: 03.65.Bz, 03.65.Sq, 03.65.Fd 


\section{INTRODUCTION}

Ever since the beginnings of quantum mechanics, physical systems have been considered which are composed of a quantum mechanical sector plus another sector described in classical terms [1]. For instance, this issue is central in the quantum theory of the measurement when the apparatus is treated classically. The same situation appears also at a less fundamental level. There are many systems in the literature which are routinely treated using a mixed quantum-classical description even if, as far as we know, they are well accounted for by quantum mechanics. Molecular theory or quantum optics are just two instances of this. The mixed description is used as a convenient approximation which greatly simplifies the treatment of such systems. In other cases some degrees of freedom are treated classically because no complete quantum theory exist for them. A typical example is the coupling of matter to gravity. In this case it is standard to use a mean field treatment (called semiclassical gravity) where a classical gravitational field obeys Einstein equations using as source the expectation value of the energy-momentum tensor of the quantum matter fields [2]. When used in early universe cosmology, this approach leads to universes which are much too uniform as compared to present observations [3]. This has been attributed [4] to the fact that the mean field approach misses the secondary quantum fluctuations induced on the classical gravitational field by its coupling to the quantum matter fields, the socalled quantum backreaction [5]. Of course, in a full quantum treatment, the gravitational field would present their own primary quantum fluctuations. Such a treatment has not been pursued because of the lack of a renormalizable quantum theory of gravitation. In principle one would expect that the renormalizability problem would be less severe when the gravitational field is classical although with quantum backreaction, however, Ref. [6] shows that very likely this will not be the case. There, it is shown that removing the primary quantum fluctuations of just one sector makes a formerly renormalizable theory into a non renormalizable one.

The problem of mixing classical and quantum degrees of freedom has been addressed by many authors from different points of view. A very incomplete list is [4 [13]. There is no generally accepted definition of what is meant by a classical-quantum system. This is natural since, as far as we know, no such system exits in nature. In the present work a "quantum-classical mixing" will mean some limit case of a quantum system. The issue that we want to study is whether such a limit can actually be taken in a way which is universal and internally consistent. Universal here refers to the existence of a well-defined set of rules to be applied to any quantum-quantum system to obtain its classical-quantum version. Since our mixed systems are just degenerated cases of quantum systems, no new universal parameters should be introduced and thus our conclusions do not directly apply to approaches such as that in [13].

As is well known, the Poisson bracket, which governs the classical dynamics, can be obtained as a limit of the quantum commutator by means of the Wigner transformation (see section [II). Such classical limit preserves a number of mathematical properties of the original quantum commutator and this makes the classical dynamics internally consistent. By internally consistent we mean that the classical dynamics does not give any clue that it is just an approximation since it displays all the correct properties that one would expect (see below in this section). Let us now consider a quantum system with two subsystems or sectors, 
which in general will be mutually interacting. One can ask whether it is possible to take the classical limit in just one of the sectors and still have an internally consistent dynamics for the resulting mixed quantum-classical system. After some definitions, this becomes a mathematical problem with some physical input which will be called the semiquantization problem here. As noted above, quantum-classical mixed systems exists abundantly in the literature, where they are understood as approximations to a fully quantum dynamics. They are not meant to be consistent so they are not under debate here.

Let us clarify in what sense classical mechanics is a consistent limit of the quantum mechanics and, in passing, introduce some notation. It is a common feature of both classical and quantum mechanics that the dynamics can be described in Heisenberg picture by an evolution equation of the form

$$
\frac{d A}{d t}=(A, H)+\frac{\partial A}{\partial t}
$$

where, $t$ is the time, $A$ is an arbitrary observable and $H$ is the Hamiltonian of the system. The term $\frac{\partial A}{\partial t}$ takes into account the intrinsic time dependence of $A$. On the other hand, the term $(A, H)$ describes the dynamic evolution of $A$. In the quantum case the observables are self-adjoint operators in a Hilbert space and the bracket $($,$) is essentially the commutator.$ In the classical case the observables are real functions on the phase space and the dynamical bracket is the Poisson bracket:

$$
\begin{aligned}
(A, B)_{q} & =\frac{1}{i \hbar}[A, B]=\frac{1}{i \hbar}(A B-B A) \\
(A, B)_{c} & =\{A, B\}=\sum_{i}\left(\frac{\partial A}{\partial x_{i}} \frac{\partial B}{\partial k_{i}}-\frac{\partial A}{\partial k_{i}} \frac{\partial B}{\partial x_{i}}\right) .
\end{aligned}
$$

Mathematically, the quantum and classical brackets have a number of remarkable properties. First, they are universal in the sense that they are independent of the particular dynamics. The latter is specified by the Hamiltonian which in principle can be any observable of the system. Second, they are Lie brackets, that is, they are linear, antisymmetric and satisfy the Jacobi identity:

$$
\begin{aligned}
& (A, B)=-(B, A), \\
& ((A, B), C)+((B, C), A)+((C, A), B)=0 .
\end{aligned}
$$

The antisymmetry of the bracket ensures that time independent Hamiltonians are conserved. The linearity guarantees that if $A(t)$ and $B(t)$ are two observables which only have dynamical evolution (i.e., without intrinsic time dependence), and $a$ and $b$ are two real constants, the observable $C_{1}(t)=a A(t)+b B(t)$ is also free of intrinsic time dependence. Likewise, the Jacobi identity ensures that the observable $C_{2}(t)=(A(t), B(t))$ also evolves dynamically only:

$$
\frac{d C_{2}}{d t}=\left(\frac{d A}{d t}, B\right)+\left(A, \frac{d B}{d t}\right)=((A, H), B)+(A,(B, H))=((A, B), H)=\left(C_{2}, H\right) .
$$

The third equality requires the Jacobi identity since $A, B$ and $H$ can be arbitrary. In particular, this property ensures the preservation of the canonical relations among canonical 
variables. Third, for any Hamiltonian, the dynamic evolution operator $(, H)$ is a derivation, that is, satisfies Leibniz's rule:

$$
(A B, H)=(A, H) B+A(B, H) .
$$

Being a derivation guarantees that the product of observables is consistent with time evolution, i.e., the observable $C_{3}(t)=A(t) B(t)$ is free of intrinsic time dependence if $A$ and $B$ are. In particular, this ensures that the commutation relations among canonical variables are preserved. (Note that commutation relations here means the commutator, which may or may not coincide with the dynamical Lie bracket.) Fourth, the brackets are such that the reality or hermiticity conditions on the observables (in the classical or quantum cases respectively) are preserved by time evolution.

Giving up these properties would imply that some of the previous constructions are not preserved by time evolution and this would introduce an intrinsic time dependence in the dynamics. Note that this is different from the question of whether the dynamics is conservative or not; a non conservative Hamiltonian $H(t)$ introduces a privileged origin of time in the dynamics but then the Hamiltonian $H^{\prime}(t)=H(t-\tau)$ defines a dynamics which is precisely the same as before except that the time is shifted by $\tau$, provided that the dynamical bracket has all the properties noted above. In the absence of any of these properties there would be a universal privileged time, universal meaning independent of the particular Hamiltonian [10]. Three further important remarks are, first, that the equivalence between Schrödinger and Heisenberg pictures can only be proved if the bracket is a derivation, since it requires that the product of observables be preserved by time evolution, second, when the bracket is a derivation, c-number observables are automatically free of dynamic evolution (since $(1, H)=\left(1^{2}, H\right)=2(1, H)=0$, the second equality coming from Leibniz rule), otherwise this property requires an independent postulate, and third, the Lie bracket property is essential if one wants the dynamical system to carry representations of symmetry groups of transformations using the observables as infinitesimal generators. The operator $(, H)$ is just a particular case corresponding to the group of time translations, hence generalizing what we have already said to other transformation groups, in the absence of the Lie bracket property the dynamics would introduce intrinsic violations of rotational invariance, etc.

In [10] a study of the semiquantization problem was carried out making two natural assumptions, first that the semiquantized theory should enjoy all mathematical properties common to both quantum and classical dynamics and second that when the two sectors are decoupled they should evolve as if they were isolated, according to their usual quantum or classical dynamics. More precisely it was required the existence of a Heisenberg picture, a canonical structure plus the condition that the product of two observables were preserved by the time evolution. It was found that under these assumptions the only consistent dynamics are either purely quantum or purely classical. It was also found that removing the canonical structure condition allows other dynamics but they are trivial in the sense that the classical variables do not inherit fluctuations from their coupling to the quantum sector, that is, there is no quantum backreaction on the classical sector. This is the case of the semiclassical dynamics commented above, where the classical variables are coupled to the expectation values of the relevant quantum observables.

In the present work, the consistency of a universal semiquantization is studied assuming only a canonical structure or assuming only physical positivity of the resulting theory. We 
consider the simplest systems such as those described by position-momentum pairs or field theories of real scalar fields. In section II we study some existent proposals of the universal type to the semiquantization problem and show that they fail to be consistent. In section III, we find that any universal canonical semiquantization fails to fulfill some natural requirements when the two sectors are decoupled. In section $\mathbb{E}$ it is found that the requirement of physical positivity of the semiquantum theory prevents the existence of quantum backreaction or even the coupling among the quantum and classical sectors. Section $\nabla$ summarizes our conclusions.

\section{THE QUANTUM-CLASSICAL BRACKET}

The general setting is as follows. There is one quantum sector and one classical sector. We will consider only systems which are described by conjugate canonical variables of the type position and momentum, that is, Hilbert spaces of the form $\mathrm{L}^{2}\left(\mathrm{R}^{n}\right)$ in the quantum case. The observables are formed out of the classical canonical variables $x_{i}, k_{i}, i=1, \ldots, n_{c}$ and the quantum ones $q_{i}, p_{i}, i=1, \ldots, n_{q}$. Therefore, they are functions defined on the phase space of the classical sector which take values on operators on the Hilbert space of the quantum sector. The classical variables are commuting numbers whereas $\left[q_{i}, q_{j}\right]=\left[p_{i}, p_{j}\right]=0,\left[q_{i}, p_{j}\right]=i \hbar \delta_{i j}$, as usual. The standard proposal for the quantum-classical bracket is

$$
(A, B)_{s}=(A, B)_{q}+\frac{1}{2}\left((A, B)_{c}-(B, A)_{c}\right)
$$

(The Poisson bracket $\{A, B\}$ is defined by eq. (2) also when $A$ and $B$ are non commuting quantities.) This dynamical bracket has been proposed by various authors [4,8, 12] starting from different considerations. It should be noted, however, that [4] uses a Schrödinger picture and so this bracket is used only to evolve the density matrix. This will be discussed further in section [V]. The bracket of [5], $(A, B)_{s}^{\prime}=(A, B)_{q}+(A, B)_{c}$, is similar except that it is not antisymmetric. In [12 a Wigner representation is chosen for the quantum operators.

This bracket can be obtained as follows. Let us start from a fully quantum system with two sectors. The Hilbert space will be $\mathcal{H}_{q} \otimes \mathcal{H}_{c}$ with $\mathcal{H}_{q, c}=\mathrm{L}^{2}\left(\mathrm{R}^{n_{q, c}}\right)$. In order to take a classical limit later, let us apply a Wigner transformation to the sector $\mathcal{H}_{c}$ :

$$
A\left(x, k ; \hbar_{c}\right)=\int d^{n_{c}} y e^{-i y \cdot k / \hbar_{c}}\left\langle x+\frac{1}{2} y|\hat{A}| x-\frac{1}{2} y\right\rangle .
$$

Here $\hat{A}$ is the original operator on the full Hilbert space $\mathcal{H}_{q} \otimes \mathcal{H}_{c} . \quad|x\rangle$ is a basis state with well-defined position in the space $\mathcal{H}_{c}$ only. Therefore, $A$ is an operator on $\mathcal{H}_{q}$ and a function on the phase space spanned by $x_{i}$ and $k_{i}, i=1, \ldots, n_{c}$. This transformation can be inverted so that $\hat{A}$ can be recovered from $A$, thus $A$ is a faithful representation of $\hat{A}$. The representation will depend on the positive parameter $\hbar_{c}$ which is entirely arbitrary.

The Wigner transformation naturally defines a product among functions on the phase space, namely (with obvious notation) $A * B$ is defined as the Wigner representation of $\hat{A} \hat{B}$. Of course, if $A, B$ are regarded as $\hbar_{c}$-independent functions, the operation represented by $*$ will depend on $\hbar_{c}$ explicitly. The commutator $[\hat{A}, \hat{B}]$ is represented by $[A, B]_{*}=A * B-B * A$ and so the fully quantum dynamical bracket is represented by $\frac{1}{i \hbar}[A, B]_{*}$ : 


$$
(\hat{A}, \hat{B})_{q}=\frac{1}{i \hbar}[\hat{A}, \hat{B}] \rightarrow \frac{1}{i \hbar}[A, B]_{*}
$$

In order to obtain the dynamical bracket of the mixed quantum-classical system, it remains to take the classical limit in the sector $\mathcal{H}_{c}$. This can be done using the identity

$$
e^{i x \cdot k / \hbar_{c}}=\left(2 \pi \hbar_{c}\right)^{n_{c}} e^{i \hbar_{c} \partial_{x} \cdot \partial_{k}} \delta(x) \delta(k)
$$

which allows to express the product $*$ as

$$
(A * B)(x, k)=e^{\frac{1}{2} i \hbar_{c}\left(\partial_{x}^{(A)} \cdot \partial_{k}^{(B)}-\partial_{k}^{(A)} \cdot \partial_{x}^{(B)}\right)} A(x, k) B(x, k) .
$$

Here, $\partial_{x}^{(A)}$ means derivative of the $x$ dependence in $A$ only, etc. This formula is convenient to study the limit of small $\hbar_{c}$. An expansion in powers of $\hbar_{c}$ gives

$$
A * B=A B+\frac{i \hbar_{c}}{2}\{A, B\}+O\left(\hbar_{c}^{2}\right) .
$$

Therefore, the dynamical bracket takes the form

$$
\frac{1}{i \hbar}[A, B]_{*}=\frac{1}{i \hbar}[A, B]+\frac{1}{2} \frac{\hbar_{c}}{\hbar}(\{A, B\}-\{B, A\})+\frac{\hbar_{c}}{\hbar} O\left(\hbar_{c}\right) .
$$

Taking now $\hbar_{c}=\hbar$ and neglecting terms of $O(\hbar)$ one gets

$$
\frac{1}{i \hbar}[A, B]+\frac{1}{2}(\{A, B\}-\{B, A\})
$$

which is just the quantum-classical bracket $(,)_{s}$ defined in eq. (6). The idea would be that neglecting higher order terms in $\hbar_{c}$ corresponds to the classical limit in the sector $\mathcal{H}_{c}$. In fact, when there is no quantum sector present, $n_{q}=0, A$ and $B$ are commuting quantities and the limit $\hbar=\hbar_{c} \rightarrow 0$ of $\frac{1}{i \hbar}[A, B]_{*}$ is well-defined and gives the Poisson bracket. On the other hand, if there is no classical sector, $n_{c}=0$, all terms containing $\hbar_{c}$ vanish (cf. eq. (10)) and the prescription reproduces the usual quantum commutator.

The construction of this bracket is somewhat tricky and in fact it does not define a consistent coupling among the classical and quantum sectors. As already noted, it is not a derivation (it does not satisfies Leibniz rule). Even if one does not insist on this requirement, the bracket $(,)_{s}$ does not define a canonical structure because it fails to fulfill the Jacobi identity. This is readily checked by taking three observables $A=q x, B=q p x$ and $C=p k^{2}$, where $q, p$ are position and momentum variables of a one dimensional quantum subsystem, and $x, k$ refer to the position and momentum of the classical subsystem, also one dimensional. By direct computation one finds

$$
\left((A, B)_{s}, C\right)_{s}+\left((B, C)_{s}, A\right)_{s}+\left((C, A)_{s}, B\right)_{s}=-\frac{1}{2}(i \hbar)^{2} .
$$

(In order to show that the Jacobi identity is violated, it is necessary to use at least two cubic operators. The identity is preserved if all the operators involved are at most quadratic in $x, k, q$ and $p$. Also, for the identity to fail at least two of the operators should be of mixed quantum-classical type.) 
Since the product $A * B$ is just a (faithful) representation of the ordinary product of operators, it is associative and thus the corresponding commutator $[A, B]_{*}$ satisfies the Jacobi identity for any value of $\hbar_{c}$. The violation of the Jacobi identity in $(,)_{s}$ comes from the truncation of the commutator at $O(\hbar)$ after taking $\hbar_{c}=\hbar$. Expanding the exact (untruncated) commutator in powers of $\hbar_{c}$ one finds

$$
[A, B]_{*}=\mathcal{C}_{0}(A, B)+\hbar_{c} \mathcal{C}_{1}(A, B)+\hbar_{c}^{2} \mathcal{C}_{2}(A, B)+\cdots
$$

The coefficients $\mathcal{C}_{n}$ are independent of $\hbar_{c}$ by definition and can be computed using eq. (10). In particular, the coefficients $\mathcal{C}_{0}$ and $\mathcal{C}_{1}$ can be read off from eq. (12), being the commutator and the Poisson bracket respectively. The Jacobi identity then yields a separate identity for each power of $\hbar_{c}$

$$
\begin{aligned}
& 0=\mathcal{C}_{0}\left(\mathcal{C}_{0}(A, B), C\right)+\text { c.p. } \\
& 0=\mathcal{C}_{0}\left(\mathcal{C}_{1}(A, B), C\right)+\mathcal{C}_{1}\left(\mathcal{C}_{0}(A, B), C\right)+\text { c.p. } \\
& 0=\mathcal{C}_{0}\left(\mathcal{C}_{2}(A, B), C\right)+\mathcal{C}_{1}\left(\mathcal{C}_{1}(A, B), C\right)+\mathcal{C}_{2}\left(\mathcal{C}_{0}(A, B), C\right)+\text { c.p. }
\end{aligned}
$$

(Where c.p. stands for cyclic permutations of $A, B$, and $C$.) When there is just one quantum sector, the only non vanishing coefficient is $\mathcal{C}_{0}$ and the first equation yields the Jacobi identity for the commutator. On the other hand, when there is just a classical sector, $\mathcal{C}_{0}$ vanishes and the third equation yields the Jacobi identity for the Poisson bracket (of commuting quantities). If there are two sectors $\mathcal{C}_{0}$ no longer vanishes and keeping only $\mathcal{C}_{0}+\hbar_{c} \mathcal{C}_{1}$, as in $(,)_{s}$, violates the Jacobi identity at $O\left(\hbar_{c}{ }^{2}\right)$. As a rule, the operation of keeping only the leading order in the expansion preserves the Jacobi identity since this can be seen as a limit case. In the purely quantum case the leading order is $\mathcal{C}_{0}$ and in the purely classical case it is $\mathcal{C}_{1}$. However, in general, working with the series truncated at $O\left(\hbar_{c}^{n}\right)$ preserves the Jacobi identity only modulo $O\left(\hbar_{c}^{n}\right)$.

Contrary to the opinion expressed in Ref. [12], we think a dynamics leading to the bracket in eq. (6) cannot be consistent. In our opinion, it is clear that any dynamics in a Heisenberg picture necessarily defines a concrete dynamical bracket and a consistent dynamics can only yield a consistent bracket. Therefore, stating that the resulting dynamical bracket is "naive" or "simplistic" [12 does not solve the consistency problem. As follows from our previous discussion, the bracket $[,]_{*}$ is indeed perfectly consistent, so the bracket $(,)_{s}$ would enjoy the Jacobi identity if it were a true limit case of $[,]_{*}$, but it is not. It is simply an arbitrary prescription. This fact, as well as the fact that the Jacobi identity is a non linear relation, accounts for the failure of that bracket to be consistent.

This is a good place to illustrate what we mean by an internally consistent semiquantization. The semiquantization given by $(,)_{s}$ is not internally consistent since checking the Lie bracket property one finds that it is not preserved, therefore it can be concluded, without using external information, that something is missing and that $(,)_{s}$ is intrinsically an approximation. As discussed above, a non-Lie dynamical bracket introduces violations of symmetries and can have a limited range of applicability only.

Since $(,)_{s}$ violates Jacobi at second order in $\hbar_{c}$, it would make sense to add the second order term $\mathcal{C}_{2}$, so that Jacobi is preserved. However, new violations would appear at higher orders and, in addition, the classical sector would be less "classical". It is to be expected that a systematic correction of the bracket in order to exactly fulfill the Jacobi identity 
would end up in a quantum-quantum system (except, may be if only particular subclasses of observables are involved). At this point we can recall that a similar conclusion was reached by De Witt long ago [7]. He showed that consistency with the uncertainty principle in the quantum sector, requires to introduce systematic corrections in the system in such a way that one ends up with a fully quantum system in both sectors. Of course, there is no problem in having a mixed system if the two sectors are never coupled, but in general, only fully classical or fully quantum dynamics are consistent. His construction pursued to obtain a consistent action functional to describe the mixed system thus effectively implying a canonical structure.

\section{OBSTRUCTIONS TO A CANONICAL SEMIQUANTIZATION}

The semiquantization problem, that is, the construction of a consistent dynamics for a mixed quantum-classical system, reminds the quantization problem. The quantization problem in its most naive form consists in associating to each function $A(x, k)$ on a classical phase space an operator $\hat{A}=A(q, p)$ in $\mathrm{L}^{2}\left(\mathrm{R}^{n}\right)$ using the quantization rules $x_{i} \rightarrow q_{i}, k_{i} \rightarrow p_{i}$ and $1 \rightarrow I$ (the identity operator) and choosing the ordering of the operators in such a way that $\{A, B\} \rightarrow(i \hbar)^{-1}[\hat{A}, \hat{B}]$. As is known, the quantization problem posed in this form does not have a solution [14]; for arbitrary functions there is no way to choose the order of the operators so that the Poisson bracket goes into commutator. The semiquantization problem can also be seen as a problem of ordering of operators since the trouble comes because $\{A, B\}$ fails to fulfill the Jacobi identity when $A$ and $B$ are not commuting. As we have seen, the naive antisymmetrization implied by $(,)_{s}$ is insufficient to produce a Lie bracket in general. The naive quantization problem has a solution when restricted to the subspace of quadratic operators (namely, using $x k \rightarrow \frac{1}{2}(q p+p q)$ ) and likewise $(,)_{s}$ is a Lie bracket when restricted to the subspace of at most quadratic operators. This is because in that case the coefficients $\mathcal{C}_{n}$ vanish for $n \geq 2$.

In order to solve the semiquantization problem, we could start trying different combinations of commutators and Poisson brackets, or equivalently different orderings for the operators. Instead of that, we will show that under very general conditions the problem does not have a solution within the canonical framework. This puts a strong constraint on the kind of semiquantizations one should look for.

Let $\mathcal{A}_{c}$ be the set of classical observables, i.e., real functions on the phase space of the classical sector, and $\mathcal{A}_{q}$ be set of quantum observables, operators on the Hilbert space of the quantum sector. The full set of observables is $\mathcal{A}=\mathcal{A}_{c} \otimes \mathcal{A}_{q}$, so a general observable will be of the form $A=\sum_{i j} C_{i} Q_{j}$ where $C_{i}$ and $Q_{j}$ are purely classical and purely quantum observables, respectively. Let $($, ) denote the semiquantum dynamical bracket, which will be assumed to be a Lie bracket. We will consider dynamics satisfying the following postulates

$$
\left(C Q, C^{\prime}\right)=\left(C, C^{\prime}\right)_{c} Q, \quad\left(C Q, Q^{\prime}\right)=\left(Q, Q^{\prime}\right)_{q} C
$$

for arbitrary purely classical observables $C, C^{\prime}$ and arbitrary purely quantum observables $Q, Q^{\prime}$. These postulates can be justified as follows. In a system formed by two quantum subsystems, the observables of one sector commute those of the other sector and so $\left[A_{1} A_{2}, A_{1}^{\prime}\right]=\left[A_{1}, A_{1}^{\prime}\right] A_{2}$ for arbitrary $A_{1}, A_{1}^{\prime}$ in one sector and $A_{2}$ in the other sector. If 
the quantum-classical system is a limit of the quantum-quantum one obtains the above postulates. Note that the postulates are free of any ordering problem. For another argument, consider that the Hamiltonian of the semiquantum system is of the form $H=C^{\prime}+Q^{\prime}$. Since both sectors are not coupled by an interaction term, each sector should evolve separately as if it were isolated. This implies that when the Hamiltonian is purely classical, a classical observable should evolve classically and furthermore, a quantum observable must not evolve. Reversing the roles of quantum and classical, and putting this in infinitesimal form it follows that

$$
\left(C, C^{\prime}\right)=\left(C, C^{\prime}\right)_{c}, \quad\left(Q, Q^{\prime}\right)=\left(Q, Q^{\prime}\right)_{q}, \quad(Q, C)=0
$$

These relations are weaker than our postulates. On the other hand, our postulates can be derived from these ones if in addition it is assumed that $(, C)$ and $(, Q)$ should be derivations. By themselves the axioms in eqs. (18) are too weak to sufficiently constrain the form of the bracket. In order to be able to draw definite conclusions we will make a stronger assumption which is equivalent to our postulates in eqs. (17) . Namely, we demand that when the Hamiltonian is classical, an observable $Q C$ should evolve into $Q C(t)$ where $C(t)$ is the classical evolution of $C$. In infinitesimal form this yields the first postulate. The second postulate follows similarly. This constraint on the form of the evolution of $Q C$ is automatically satisfied if the quantum-classical system derives as a limit from a quantumquantum system and therefore it is a very natural requirement. Note that there is an implicit assumption of universality in the argument, i.e. the dynamical bracket should be the same for all Hamiltonians and any observable can be a Hamiltonian. Then we can state the following theorem:

Theorem 1: Let $\mathcal{A}=\mathcal{A}_{c} \otimes \mathcal{A}_{q}$ be of the position-momentum type in both sectors. Then, no Lie bracket $($, ) in $\mathcal{A}$ can fulfill the axioms

$$
(A, C)=(A, C)_{c}, \quad(A, Q)=(A, Q)_{q}
$$

for all $C \in \mathcal{A}_{c}, Q \in \mathcal{A}_{q}$ and $A \in \mathcal{A}$.

Note that, because all observables are of the form $\sum_{i j} C_{i} Q_{j}$, these axioms are equivalent to those in eq. (17). The bracket $(,)_{s}$ satisfies these axioms and thus it is a particular case.

It should be remarked that the theorem only applies to systems described by position and momentum conjugate variables. Other quantum-classical mixtures, e.g., a quantum sector with a finite dimensional Hilbert space such as a spin system plus some classical sector, are not directly ruled out by this theorem. Also, the incompatibility refers to a semiquantization of the complete class of observables. As noted above, the bracket $(,)_{s}$, which fulfills our postulates, is a Lie bracket in the restricted subclass of observables which are at most quadratic in $q, p, x, k$.

What follows in this section is devoted to the proof of this theorem.

In order to prove the incompatibility stated in the theorem, let us assume that $($,$) is a$ Lie bracket which satisfies our axioms. It will be sufficient to consider a system with a onedimensional quantum sector and a one-dimensional classical sector. Also, in what follows we will take $\hbar=1$ since keeping $\hbar$ variable (but strictly positive) does not add anything to the proof. Let us consider the set of observables

$$
e_{r}=e_{r}^{c} e_{r}^{q}, \quad e_{r}^{c}=e^{i k_{r} x-i x_{r} k}, \quad e_{r}^{q}=e^{i p_{r} q-i q_{r} p} .
$$


Where $x_{r}, k_{r}, q_{r}$ and $p_{r}$ are arbitrary real numbers and $x, k, q$ and $p$ are the dynamical variables. The observables of the form $e_{r}^{c}$ form a basis of $\mathcal{A}_{c}$ and those of the form $e_{r}^{q}$ form a basis of $\mathcal{A}_{q}$. This latter statement is more clearly seen by using the form $e_{r}^{q}=$ $e^{-\frac{1}{2} i p_{r} q_{r}} e^{i p_{r} q} e^{-i q_{r} p}$ since $e^{i p_{r} q}$ and $e^{-i q_{r} p}$ are basis of the operators which are functions of $q$ and $p$ respectively and any operator in $\mathrm{L}^{2}(\mathrm{R})$ can be normal ordered putting the $q$ at the left of the $p$. Therefore, $e_{r}$ defines a (linear) basis of $\mathcal{A}$. Let us see that the bracket can be determined up to a c-number function. Using the postulates, it is immediate that

$$
\left(e_{r}, x\right)=i x_{r} e_{r}, \quad\left(e_{r}, k\right)=i k_{r} e_{r}, \quad\left(e_{r}, q\right)=i q_{r} e_{r}, \quad\left(e_{r}, p\right)=i p_{r} e_{r} .
$$

The Jacobi identity can be expressed as

$$
\delta_{C}(A, B)=\left(\delta_{C} A, B\right)+\left(A, \delta_{C} B\right), \quad \delta_{C}:=(, C),
$$

for arbitrary $A, B$ and $C$. Therefore, the Jacobi identity requires

$$
\begin{gathered}
\left(\left(e_{r}, e_{s}\right), x\right)=i\left(x_{r}+x_{s}\right)\left(e_{r}, e_{s}\right), \quad\left(\left(e_{r}, e_{s}\right), k\right)=i\left(k_{r}+k_{s}\right)\left(e_{r}, e_{s}\right), \\
\left(\left(e_{r}, e_{s}\right), q\right)=i\left(q_{r}+q_{s}\right)\left(e_{r}, e_{s}\right), \quad\left(\left(e_{r}, e_{s}\right), p\right)=i\left(p_{r}+p_{s}\right)\left(e_{r}, e_{s}\right) .
\end{gathered}
$$

On the other hand, $\left(e_{r}, e_{s}\right)$ will be a linear combination of the $e_{t}$ and the previous equations imply that $x_{t}=x_{r}+x_{s}, k_{t}=k_{r}+k_{s}, q_{t}=q_{r}+q_{s}$ and $p_{t}=p_{r}+p_{s}$. In summary,

$$
\begin{aligned}
\left(e_{r}, e_{s}\right) & =F\left(q_{r}, p_{r}, x_{r}, k_{r} ; q_{s}, p_{s}, x_{s}, k_{s}\right) e^{i\left(k_{r}+k_{s}\right) x-i\left(x_{r}+x_{s}\right) k+i\left(p_{r}+p_{s}\right) q-i\left(q_{r}+q_{s}\right) p} \\
& :=F_{r s} e_{r+s} .
\end{aligned}
$$

Here $F$ is some real function which depends on the particular bracket only. The postulates are consistent with this form and correspond to

$$
\begin{aligned}
& F\left(q_{r}, p_{r}, x_{r}, k_{r} ; 0,0, x_{s}, k_{s}\right):=F_{r s}^{c}=v_{r s}, \\
& F\left(q_{r}, p_{r}, x_{r}, k_{r} ; q_{s}, p_{s}, 0,0\right):=F_{r s}^{q}=2 \sin \left(\frac{u_{r s}}{2}\right),
\end{aligned}
$$

where we have introduced the variables

$$
u_{r s}=p_{r} q_{s}-q_{r} p_{s}, \quad v_{r s}=k_{r} x_{s}-x_{r} k_{s} .
$$

The functions $F^{c}$ and $F^{q}$ come from computing $\left(e_{r}^{c}, e_{s}^{c}\right)_{c}$ and $\left(e_{r}^{q}, e_{s}^{q}\right)_{q}$, respectively.

Up to now we have imposed the Jacobi identity only when one of the operators is $x, k$, $q$ or $p$. The full Jacobi identity follows from considering $\left(\left(e_{r}, e_{s}\right), e_{t}\right)$. It is immediate that the Lie bracket property can be expressed as

$$
\begin{aligned}
& F_{r s}=-F_{s r}, \\
& F_{r s} F_{r+s, t}+F_{s t} F_{s+t, r}+F_{t r} F_{t+r, s}=0 .
\end{aligned}
$$

It is interesting to note that eqs. (27) are valid in a purely classical or purely quantum case. So $F^{c}$ and $F^{q}$ (and in fact, their generalization for any number of degrees of freedom) are solutions of those relations. The quantum-classical bracket of the previous section gives

$$
F_{r s}^{s}=2 \sin \left(\frac{u_{r s}}{2}\right)+v_{r s} \cos \left(\frac{u_{r s}}{2}\right) .
$$


This bracket comes from a classical expansion (but not a limit) around the quantum-quantum case:

$$
\begin{aligned}
F_{r s}^{q q} & =2 \sin \left(\frac{u_{r s}}{2}\right) \cos \left(\frac{v_{r s}}{2}\right)+2 \cos \left(\frac{u_{r s}}{2}\right) \sin \left(\frac{v_{r s}}{2}\right) \\
& =2 \sin \left(\frac{u_{r s}}{2}+\frac{v_{r s}}{2}\right),
\end{aligned}
$$

(which, of course, is equivalent to a two dimensional quantum case). $F^{s}$ satisfies the axioms, eqs. (25), but fails to fulfill the Jacobi identity, second eq. (27). Let us show that the eqs. (25) and (27) are, in fact, incompatible. As proven in appendix A, these equations imply that $F$ can only depend on the combinations $u$ and $v$ introduced above, that is

$$
F_{r s}=F\left(u_{r s}, v_{r s}\right) \text {. }
$$

This follows only from the Lie bracket property of $F$ and the fact that the boundary conditions, eqs. (25), depend also on $u$ and $v$. Using eq. (30), the postulates become

$$
F(u, 0)=2 \sin (u / 2), \quad F(0, v)=v
$$

and the Lie bracket conditions become

$$
\begin{aligned}
& F(u, v)=-F(-u,-v), \\
& F\left(u_{r s}, v_{r s}\right) F\left(u_{r t}+u_{s t}, v_{r t}+v_{s t}\right)+\text { c.p. }=0 .
\end{aligned}
$$

As proven in appendix A, these Lie bracket conditions plus $\partial_{u} F(0,0)=\partial_{v} F(0,0)=1$, only admit the solutions

$$
F(u, v)=\frac{1}{h} \sin (h u+h v)
$$

( $h$ being and arbitrary constant) or the degenerated case $F(u, v)=u+v$, which cannot accommodate the two postulates, eqs. (31), for any value of $h$. This implies that the function $F$ is only consistent with Jacobi if it is purely quantum $F(x)=2 \sin (x / 2)$ or purely classical $F(x)=x$, but does not admit mixed types. This completes the proof.

The proof of the incompatibility of the axioms can also be done by an alternative method which is constructive (but requires to perform symbolic calculations with the help of a computer). We will devote the reminder of this section to discuss this method.

Let us consider a basis of the space $\mathcal{A}$ with observables of the form $q^{r} p^{s} x^{t} k^{\ell}$, where $r, s, t, \ell=0,1, \ldots$ (The order $q p x k$ will be taken as the canonical order of these variables.) Each basis element can be assigned a degree given by $n=r+s+t+\ell$. Let us use the notation $C_{n}, Q_{n}, M_{n}$ and $A_{n}$ to refer to basis elements of degree $n$ which are a purely classical (i.e., $r=s=0)$, purely quantum $(t=\ell=0)$, mixed quantum-classical $(r+s>0$ and $t+\ell>0)$ and arbitrary, respectively. Using the axioms, the brackets of each pair of basis elements can be worked out except those of the form $\left(M_{n}, M_{n^{\prime}}\right)$ (where necessarily $n, n^{\prime} \geq 2$ ). Using the Jacobi identity, as in eq. (22), for $\xi=q, p, x, k$, yields

$$
\begin{aligned}
\left(\left(M_{n}, M_{n^{\prime}}\right), \xi\right) & =\left(\left(M_{n}, \xi\right), M_{n^{\prime}}\right)+\left(M_{n},\left(M_{n^{\prime}}, \xi\right)\right) \\
& =\left(M_{n-1}, M_{n^{\prime}}\right)+\left(M_{n}, M_{n^{\prime}-1}\right) .
\end{aligned}
$$


Since the knowledge of $(A, \xi)$ for $\xi=q, p, x, k$ determines $A$ completely up to an additive cnumber constant, this relation allows to determine $\left(M_{n}, M_{n^{\prime}}\right)$ by induction up to an additive constant. Such constants play a similar role as the function $F$ in the plain wave basis used above. Their number increases rapidly with $n$ and $n^{\prime}$.

We proceed by selecting values for these constants so that the Jacobi identity is fulfilled for arbitrary operators, if possible. Because the Jacobi identity is trivial unless two of the operators involved are of mixed type, only the case $\langle M, M, A\rangle$ gives information on the constants. At step 1 we consider the brackets of the form $\left(M_{2}, M_{2}\right)$ which contain 6 unknown constants. These constants are uniquely determined imposing the Jacobi identity to the triples $\left\langle M_{2}, M_{2}, Q_{2}\right\rangle$ and $\left\langle M_{2}, M_{2}, C_{2}\right\rangle$. The Jacobi identity for $\left\langle M_{2}, M_{2}, M_{2}\right\rangle$ turns out to be fulfilled automatically. From now on, these 6 constants are fixed to their unique value. At step 2 , we consider $\left(M_{2}, M_{3}\right)$ which contain 48 unknowns. These constants are uniquely determined imposing Jacobi to $\left\langle M_{2}, M_{3}, Q_{2}\right\rangle$ and $\left\langle M_{2}, M_{3}, C_{2}\right\rangle$, and again Jacobi for $\left\langle M_{2}, A_{3}, M_{2}\right\rangle$ comes out automatically. The 48 unknowns are then fixed to their unique value. At step 3, the 100 unknowns in $\left(M_{2}, M_{4}\right)$ are fixed to their unique value which is determined from $\left\langle M_{2}, M_{4}, Q_{2}\right\rangle$ and $\left\langle M_{2}, M_{4}, C_{2}\right\rangle$, and Jacobi for $\left\langle M_{2}, A_{4}, M_{2}\right\rangle$ is automatic. All these constants are those corresponding to the bracket $(,)_{s}$ of section 11 . Finally, at step 4 the procedure breaks down. The 66 unknowns of $\left(M_{3}, M_{3}\right)$ are uniquely determined by $\left\langle M_{3}, Q_{3}, M_{2}\right\rangle$ and $\left\langle M_{3}, C_{3}, M_{2}\right\rangle$, but they turn out to be inconsistent with the Jacobi identity for $\left\langle M_{3}, M_{3}, M_{2}\right\rangle$.

\section{QUANTUM BACKREACTION AND POSITIVITY}

In this section we want to discuss obstructions to mixing quantum and classical systems not related to a canonical structure but to the requirement of positivity.

Here we no longer require the existence of a canonical structure plus a Heisenberg picture, etc. In view of this we will need a definition of what it is meant by a quantum-classical system. We will demand that the quantum variables obey to the usual quantum commutation relations and the classical variables commute. Because the quantum variables do not commute, the usual proof shows that they must be subjected to the uncertainty principle. They have primary quantum fluctuations. (They are primary because they exist even in the absence of a coupling to other degrees of freedom.) If the two sectors are coupled, the classical observables may have induced fluctuations, the so-called quantum backreaction. The two requirements of primary fluctuations in the quantum variables and commutation of the classical variables is just what we mean by "quantum-classical" mixing. We want to argue that even these weak conditions, plus another natural requirement, namely, that the mixed quantum-classical system must correspond to some limit case of a quantum-quantum system, forbid the possibility of a quantum backreaction on the classical sector. Since such secondary fluctuations are expected to appear whenever the two sectors are coupled by an interaction term, this would suggest that no consistent quantum-classical mixing exists.

The requirement that the mixed system should be a limit of a full quantum system seems weak but still has non trivial consequences: if some quantity of the quantum-quantum system is always positive, the corresponding quantity must at least be non negative in the quantumclassical system. For instance, in the purely quantum theory the variance of any observable must be non negative, i.e., $\left\langle A^{2}\right\rangle \geq\langle A\rangle^{2}$, where \langle\rangle refers to the quantum average. The equal 
sign corresponds to an observable which is free of quantum fluctuations and so it implies $\langle f(A)\rangle=f(\langle A\rangle)$ for any function $f(x)$ as well. Then the same properties must hold in the mixed quantum-classical system. Of course, they hold in the purely classical system in particular. This is equivalent to say that whatever is the measure corresponding to the fluctuations in the mixed system (induced by the quantum sector) it must be non negative.

As is well-known in quantum mechanics, if the commutator of two observables does not vanish on some quantum state vector, at least one of the observables must present quantum fluctuations in that state. Here we will need a reciprocal of this statement:

Theorem 2: In a purely quantum theory, let $\left|\psi_{0}\right\rangle$ be the normalized ground state and $A$ any observable, then i)

$$
0 \leq-\frac{i \hbar}{2}\langle[A, \dot{A}]\rangle_{\psi_{0}}
$$

and ii) when the ground state is not degenerated, the equal sign holds if and only if $A$ is free of dispersion in $\left|\psi_{0}\right\rangle$.

This can be proved as follows. Let $H$ be the Hamiltonian and $E_{0}$ the ground state energy, $H\left|\psi_{0}\right\rangle=E_{0}\left|\psi_{0}\right\rangle$, then, because $H-E_{0}$ is non negative and $A$ is self-adjoint, the operator $A\left(H-E_{0}\right) A$ is also non negative. Therefore

$$
0 \leq\left\langle A\left(H-E_{0}\right) A\right\rangle_{\psi_{0}}=-\frac{1}{2}\langle[A,[A, H]]\rangle_{\psi_{0}} .
$$

Eq. (35) follows then from the relation $i \hbar \dot{A}=[A, H]$. To show ii), assume that $A$ has no dispersion in the ground state, $\left\langle A^{2}\right\rangle_{\psi_{0}}=\langle A\rangle_{\psi_{0}}^{2}$, then $A\left|\psi_{0}\right\rangle$ is proportional to $\left|\psi_{0}\right\rangle$ and automatically $\langle[A, B]\rangle_{\psi_{0}}=0$ for any $B$. Conversely, assume that the equal sign applies in eq. (35), then $0=\left\langle A\left(H-E_{0}\right) A\right\rangle_{\psi_{0}}$, and so $A\left|\psi_{0}\right\rangle$ is another ground state. If this is not degenerated $A\left|\psi_{0}\right\rangle$ must be proportional to $\left|\psi_{0}\right\rangle$ and $A$ is free of dispersion in the ground state.

A corollary of this theorem is that when $A$ commutes with $\dot{A}, A$ cannot have quantum fluctuations in a non degenerated ground state, so in some sense it is a reciprocal of the argument leading to the uncertainty principle. It has immediate consequences to the semiquantization problem. For a mixed system at zero temperature (and so in the ground state) the classical variables $x_{i}$ and $k_{i}=\dot{x}_{i}$ will commute with each other and, being the limit of a quantum-quantum system, they will be free of quantum fluctuations. In other words, since any induced quantum fluctuations would spoil the commutativity of the classical variables, in a quantum-classical mixing there cannot be quantum backreaction on the classical sector.

It should be noted that there are actually proposals of mixed quantum-classical systems in which the classical variables commute and at the same time have secondary quantum fluctuations. Such prescriptions exists, without invalidating our conclusions above, because they violate positivity of the measure of the quantum fluctuations. That is, positive observables do not have a positive expectation value. A first example is the proposal in [4]. There the quantum-classical system is described in terms of a density matrix which depends on the quantum variables $q, p$ and the classical variables $x, k$. The evolution is described in the Schrödinger picture in the form $\dot{\rho}=(\rho, H)$ where $H$ is the Hamiltonian and $($,$) is$ the dynamical bracket. In [4 the bracket is completely determined by imposing several natural requirements; it should reduce to the commutator or Poisson bracket as particular 
cases, the evolution preserves the hermiticity and the trace of the density matrix, and it is invariant under classical canonical transformations and quantum unitary transformations. The result is again the bracket $(,)_{s}$ in eq. (6). As noted by the authors, there is, however, one essential requirement which is violated by this construction, namely, if one starts with a positive density matrix $\rho$, its positivity is not preserved by the evolution, in general. It is noteworthy that, since the observables do not evolve, this construction does not introduce any intrinsic time dependence in the dynamics. For instance, the commutator $[q, p]=i \hbar$ is automatically time independent and the classical variables always commute. As noted, when $(, H)$ is not a derivation, the Heisenberg and Schrödinger dynamics are no longer equivalent and there is no contradiction with our discussion above, which refers to the Heisenberg picture. Nevertheless, because Jacobi is not satisfied, there will be problems implementing time-independent symmetry transformations, in addition to the positivity problem.

Another proposal is that of [6]. There, it is noted that the stochastic quantization program [15 17] leads to a natural definition of a semiquantized dynamics. As it may be recalled, in the stochastic quantization approach the dynamical variables evolve in a fictitious time, the simulation time, following a stochastic differential equation, the Langevin equation, which corresponds to a particular Monte Carlo method to sample the Euclidean path integral of the system. For instance, let $S[\phi]$ be the Euclidean action of quantum field theory with $n$ scalar fields $\phi_{i}(x), i=1, \ldots, n$ in a flat four dimensional space-time. Then the functional integral with Boltzmann weight $e^{-S / \hbar}$ is correctly sampled by the equilibrium distribution of a random walk described by the following Langevin equation [16]

$$
\frac{\partial \phi_{i}(x ; \tau)}{\partial \tau}=-\frac{\delta S[\phi]}{\delta \phi_{i}(x ; \tau)}+\sqrt{\hbar} \eta_{i}(x ; \tau) .
$$

Here $\tau$ is the simulation time and $\eta_{i}(x ; \tau)$ are independent stochastic centered Gaussian variables normalized to

$$
\left\langle\left\langle\eta_{i}(x ; \tau) \eta_{i^{\prime}}\left(x^{\prime} ; \tau^{\prime}\right)\right\rangle\right\rangle=2 \delta_{i i^{\prime}} \delta\left(x-x^{\prime}\right) \delta\left(\tau-\tau^{\prime}\right) .
$$

The variables $\eta_{i}(x ; \tau)$ introduce the quantum fluctuations in the system. In their absence, the fields $\phi$ would fall into a solution of the (Euclidean) classical equations of motion, $\delta S / \delta \phi_{i}(x)=0$.

The $\hbar$ dependence of the Langevin equation suggests a natural definition for the semiquantized system [6], namely, to replace $\hbar$ by $\hbar_{i}=0,1$ where 1 corresponds to a quantum degree of freedom and 0 to a classical one. (We will use units $\hbar=1$ from now on.) The classical degrees of freedom will not have primary quantum fluctuations but, if they are coupled to the quantum sector, they will present induced secondary fluctuations. In order to see what consequences follow from such proposal, let us take the example studied in [6]. Consider a system composed by two relativistic fields with a quadratic action

$$
S\left(\phi_{1}, \phi_{2}\right)=\int d^{4} x\left(\frac{1}{2}\left(\partial \phi_{1}\right)^{2}+\frac{1}{2} m_{1}^{2} \phi_{1}^{2}+\frac{1}{2}\left(\partial \phi_{2}\right)^{2}+\frac{1}{2} m_{1}^{2} \phi_{2}^{2}+g \phi_{1} \phi_{2}\right) .
$$

Since the action is translationally invariant, it is convenient to use a momentum representation:

$$
S\left(\phi_{1}, \phi_{2}\right)=\int \frac{d^{4} k}{(2 \pi)^{4}} \frac{1}{2} \Phi^{\dagger}(k) M(k) \Phi(k),
$$


where

$$
\Phi(k)=\left(\begin{array}{c}
\tilde{\phi}_{1}(k) \\
\tilde{\phi}_{2}(k)
\end{array}\right), \quad M(k)=\left(\begin{array}{cc}
k^{2}+m_{1}^{2} & g \\
g & k^{2}+m_{2}^{2}
\end{array}\right)
$$

and $\tilde{\phi}_{i}(k)$ is the Fourier transform of $\phi_{i}(x)$. As usual, we will assume $m_{1}^{2}, m_{2}^{2}>0$ and $m_{1}^{2} m_{2}^{2}>$ $g^{2}$, so that $M(k)$ is positive definite. Because the action is quadratic, the equilibrium solution of the Langevin equation can be solved in closed form. The connected two-point function or propagator is given in momentum space by the following matrix [6]

$$
W(k)=\frac{\hbar_{2}\left(k^{2}+m_{1}^{2}\right)+\hbar_{1}\left(k^{2}+m_{2}^{2}\right)}{\left(k^{2}+m_{1}^{2}\right)+\left(k^{2}+m_{2}^{2}\right)} W_{Q}(k)+\frac{\hbar_{1}-\hbar_{2}}{\left(k^{2}+m_{1}^{2}\right)+\left(k^{2}+m_{2}^{2}\right)} \sigma_{z},
$$

where $\sigma_{z}$ refers to the Pauli matrix $\operatorname{diag}(1,-1)$, and $W_{Q}(k)$ is the inverse matrix of $M(k)$. In this formula the $\hbar_{i}$ are arbitrary non negative numbers. The propagator in $x$-space is

$$
\left\langle T \phi_{i}(y) \phi_{j}(x)\right\rangle=\int \frac{d^{4} k}{(2 \pi)^{4}} e^{-i k(y-x)} W_{i j}(k) .
$$

This Green's function is directly connected since $\left\langle\phi_{i}(x)\right\rangle=0$. It can be shown [6] that there are no connected Green's functions of three or more points, so the system is Gaussian.

From the form of $W(k)$ it follows that in the fully classical case, $\hbar_{i}=0$, the connected two-point function vanishes implying that the fields are free from fluctuations. On the other hand, in the fully quantum case, $\hbar_{i}=1, W$ is just $W_{Q}=M^{-1}$ which is the standard quantum propagator. If $\hbar_{1}=1$ and $\hbar_{2}=0$, and, in addition, $g \neq 0,\left\langle\left(\phi_{2}(x)\right)^{2}\right\rangle$ will not vanish and thus $\phi_{2}$ is subjected to induced secondary fluctuations. When $g=0$ both sectors are decoupled.

On the other hand, we can obtain the equal-time commutation relations of the fields by considering the large momentum limit of the propagator:

$$
W(k)=\frac{1}{k^{2}}\left(\begin{array}{cc}
\hbar_{1} & 0 \\
0 & \hbar_{2}
\end{array}\right)+O\left(\frac{1}{k^{4}}\right)
$$

This directly implies

$$
\delta\left(y^{0}-x^{0}\right)\left\langle\left[\phi_{i}(y), \dot{\phi}_{j}(x)\right]\right\rangle=\hbar_{i} \delta_{i j} \delta(x-y) .
$$

Therefore, if $\hbar_{1}=1$ and $\hbar_{2}=0$, the field $\phi_{2}$ will be classical, in the sense that it commutes with its conjugate momentum, even if it is subjected to quantum backreaction from the quantum field $\phi_{1}$.

We have checked that this system is a quantum-classical mixture according to our previous definition and also that there is quantum backreaction, but we still have to see if it preserves positivity. It can be shown [6] that the matrix $W(k)$ is definite positive for all momenta and arbitrary non negative $\hbar_{i}$. However, as is well known, physical positivity corresponds rather to the stronger requirement of reflection positivity in Euclidean space [18]. Since the theory is quadratic, it is sufficient to study the Lehmann representation of the propagator, which comes from inserting a complete set of eigenstates:

$$
W_{i j}(k)=\int d \mu \frac{\rho_{i j}(\mu)}{k^{2}+\mu}
$$


where the spectral density is defined as

$$
\rho_{i j}\left(q^{2}\right)=(2 \pi)^{3} \sum_{n} \delta^{4}\left(p_{n}-q\right)\left\langle 0\left|\phi_{i}(0)\right| n\right\rangle\left\langle n\left|\phi_{j}(0)\right| 0\right\rangle
$$

Reflection positivity requires $\rho(\mu) \geq 0$. For the purely quantum case we have

$$
W_{Q}(k)=\frac{P_{+}}{k^{2}+m_{+}^{2}}+\frac{P_{-}}{k^{2}+m_{-}^{2}},
$$

where $P_{ \pm}$are the two orthogonal projectors onto the normal modes, corresponding to diagonalize $M(k)$, and $m_{ \pm}^{2}=\frac{1}{2}\left(m_{1}^{2}+m_{2}^{2} \pm R\right)$ are their squared masses (with $R=\sqrt{\left(m_{1}^{2}-m_{2}^{2}\right)^{2}+4 g^{2}}$ ). For arbitrary $\hbar_{i}$ it is found

$$
W(k)=\frac{Q_{+}}{k^{2}+m_{+}^{2}}+\frac{Q_{-}}{k^{2}+m_{-}^{2}}+\frac{Q_{3}}{k^{2}+m_{3}^{2}}
$$

where $m_{3}^{2}=\frac{1}{2}\left(m_{1}^{2}+m_{2}^{2}\right)$, and

$$
\begin{aligned}
Q_{ \pm} & =\left(\frac{\hbar_{1}+\hbar_{2}}{2} \pm \frac{\left(\hbar_{1}-\hbar_{2}\right)\left(m_{1}^{2}-m_{2}^{2}\right)}{2 R}\right) P_{ \pm}, \\
Q_{3} & =\frac{\hbar_{1}-\hbar_{2}}{2}\left(\sigma_{z}-\frac{m_{1}^{2}-m_{2}^{2}}{R}\left(P_{+}-P_{-}\right)\right) .
\end{aligned}
$$

One can see that there is an extra mode, namely, $m_{3}^{2}$. Unfortunately, whereas $Q_{ \pm}$are non negative, $Q_{3}$ is not in general, since $\operatorname{tr}\left(Q_{3}\right)=0$. This means that the covariance matrix $W(k)$ is positive but not reflection positive except in the trivial cases $\hbar_{1}=\hbar_{2}$ or $g=0$. The latter case describes two non interacting sectors, and the first case corresponds to two classical sectors if $\hbar_{1}=\hbar_{2}=0$ or two quantum sectors if $\hbar_{1}=\hbar_{2}>0$. Beyond these trivial cases, this theory does not define a Hilbert space with positive definite metric, i.e., it does not define a positive physical measure, and for instance, one can construct operators with negative variance. In other words, the probabilistic interpretation (of which the classical case is a limit) breaks down. The theory must be rejected (or else work with a restricted set of observables, which in this context would be ad hoc).

Mathematically, the lack of reflection positivity is a direct consequence of the commutation relations eq. (45). In effect, as noted, the commutation relations are equivalent to eq. (44), and comparing with eq. (49) for large $k^{2}$, it follows that $\hbar_{2}=\left(Q_{+}+Q_{-}+Q_{3}\right)_{22}$. Therefore, if $\phi_{2}(x)$ is classical and so $\hbar_{2}=0$, the cancellation requires $\left(Q_{3}\right)_{22}$ to be negative. This argument can be expected to hold on general grounds. Indeed, we have the following theorem:

Theorem 3: A theory of relativistic scalar fields which is quadratic, reflection positive, translationally invariant and $\left\langle\phi_{i}\right\rangle=0$, cannot have quantum and classical sectors unless they are decoupled.

This can be proved as follows. Under the assumptions, all information on the theory is contained in the propagator or equivalently in the spectral density $\rho(\mu)$. For simplicity we consider theories with one quantum field $\phi_{1}$ and one classical field $\phi_{2}$. Being a classical field means that $\phi_{2}$ and $\dot{\phi}_{2}$ commute at equal time. Then $\left\langle T \phi_{2}(x) \phi_{2}(y)\right\rangle$ and $\left\langle T \phi_{2}(x) \dot{\phi}_{2}(y)\right\rangle$ are 
continuous regarded as functions of $t=x_{0}-y_{0}$ at $t=0$. This implies that the function $\left\langle T \phi_{2}(x) \phi_{2}(y)\right\rangle$ is continuous and with continuous first derivative at $t=0$. As a consequence its Fourier transform must be $W_{22}(k)=O\left(\frac{1}{k^{4}}\right)$ for large $k^{2}$. From the Lehmann representation, this implies that $\rho_{22}(\mu)$ must average to zero (otherwise $W_{22}(k)=O\left(\frac{1}{k^{2}}\right)$ ) and then reflection positivity requires $\rho_{22}=0$ everywhere. At this point we have already shown that the connected propagator of a classical field must vanish and so the classical field cannot have secondary quantum fluctuations. This is in agreement with our Theorem 2 above. The stronger statement in Theorem 3 comes from noting that if $\rho_{22}=0$, positivity of the matrix $\rho$ requires $\rho_{12}=\rho_{21}=0$ as well, therefore there is no mixing among the two sectors.

\section{SUMMARY AND CONCLUSIONS}

In the present work, we study the internal consistency of semiquantization schemes of the universal type. In the Introduction it was argued that the classical dynamics is an internally consistent limit of the quantum dynamics, since the Poisson bracket preserves a number of essential properties of the quantum commutator (Lie bracket property, Leibniz's rule and hermiticity). Both dynamics, quantum and classical, are of the universal type since they have a fixed dynamical bracket independent of the particular Hamiltonian. There it is also discussed what unacceptable consequences would follow by giving up any of the above mentioned properties, namely, intrinsic breaking of symmetries and lack of hermiticity.

In section 1 the standard quantum-classical dynamical bracket for a system with two sectors (each of the type position-momentum) is derived as a "limit" of the quantum-quantum bracket with the help of the Wigner representation. It is argued that such a bracket is not internally consistent, since it fails to satisfy both the Jacobi identity and the Leibniz's rule. It is pointed out that this failure is due to the fact that such a bracket is not a true limit case but rather a truncation at second order in an expansion in $\hbar$. On the contrary the classical limit (in all sectors) is a true limit since it keeps only the leading order in $\hbar$ and so it preserves both the Jacobi identity and the Leibniz's rule.

In section III the semiquantization problem in its canonical version is studied. It is pointed out the similarity of this problem with that of the naive quantization of classical systems, which is known not to have a solution for arbitrarily large spaces of observables. Both problems can be tied to the ordering problem of operators.

Roughly speaking, the canonical semiquantization problem consists in finding a Lie bracket in the algebra of observables of the mixed quantum-classical theory which interpolates between the Poisson bracket and the quantum commutator. (The Leibniz's rule, which is common to both classical and quantum dynamics, is not imposed on the semiquantum bracket, since it was already shown in [10] that such a requirement plus the Lie bracket condition only allows for purely classical or purely quantum dynamics, at least for systems of the position-momentum type.) However, in order to determine the bracket of observables of mixed type, some assumptions have to be made. Our assumptions are cast in the axioms in eq. (17). They follow either from assuming that the quantum-classical system is a limit of a quantum-quantum system or else from natural requirements on the behavior of the two sectors when they are decoupled. Such axioms are sufficiently general as to cover the case of the standard quantum-classical bracket of section 【1. Theorem 1 is the main result of section III. It states that, if both sectors are of the position-momentum type (and are non 
trivial, i.e., they are not zero dimensional) no Lie bracket exists which satisfies the axioms. In other words, under the assumptions, there is no consistent canonical semiquatization. It would be interesting to know whether the axioms allow for consistent semiquantizations when one or both sectors are not of the position-momentum type. For instance, one can consider a particle with classical position and momentum but quantum spin. It would also be interesting to know whether Theorem 1 can be adapted when the axioms are weakened to those in eq. (18), or else, to find what kind of consistent semiquantizations are obtained.

In section $[7$ the requirement of a canonical structure for the semiquantized system is dropped. It is only imposed that the variables in the quantum sector are not commuting and those in the classical sector are commuting, and further that positive observables must have positive expectation values. This latter requirement follows immediately if the mixed classical-quantum system is the limit of a quantum-quantum system.

Being non commuting, the standard argument shows that the quantum variables must have primary quantum fluctuations. In section $\mathbb{\nabla}$ a sort of reciprocal of the uncertainty principle is proven, Theorem 2, which implies that commuting variables cannot have fluctuations. Therefore, under the assumptions, any semiquantization scheme either does not have quantum backreaction on the classical sector or else breaks positivity. One example of the first possibility is semiclassical gravity. Two examples of the second possibility are discussed in section $\mathbb{}$ V. Finally a further Theorem 3 is presented which shows that, for scalar fields with quadratic Lagrangians, positivity of the theory not only forbids any quantum backreaction on the classical sector but also any coupling among the two sectors.

These negative results, contained in the three theorems proven in the text, put constraints to the form of possible semiquantization approaches, especially those of universal type. A further negative result has already been noted: quantum-classical field theories fail to be renormalizable even if their corresponding quantum-quantum version is renormalizable [6]. Nevertheless, it should be kept in mind that our conclusions only apply under the assumptions made, and so more general forms of mixed quantum-classical systems cannot be ruled out.

\section{ACKNOWLEDGMENTS}

This work is supported in part by funds provided by the Spanish DGICYT grant no. PB95-1204 and Junta de Andalucía grant no. FQM0225.

\section{APPENDIX A: PROOF OF EQS. (30) AND (33)}

In order to prove eq. (30), let us consider the Jacobi identity, eq. (27), with $x_{t}=k_{t}=0$. This yields

$$
F_{r s} F_{r+s, t}^{q}+F_{s t}^{q} F_{t+s, r}+F_{t r}^{q} F_{t+r, s}=0 .
$$

Let us recall that $F_{r s}^{q}=2 \sin \left(u_{r s} / 2\right)$, with $u_{r s}=p_{r} q_{s}-q_{r} p_{s}$. Using the antisymmetry of $F$, the relation can be written as

$$
F_{s t}^{q} F_{r, s+t}=F_{r+s, t}^{q} F_{r s}-F_{r t}^{q} F_{r+t, s} .
$$


Note that $F_{r s}, F_{r, s+t}$ and $F_{r+t, s}$ all have the same dependence on the classical variables $x_{r}, k_{r}, x_{s}, k_{s}$ and so these variables can be treated just as fixed parameters in $F$, thus we can concentrate on the dependence on the quantum sector only and use the notation $F_{r s}=$ $F\left(q_{r}, p_{r} ; q_{s}, p_{s}\right)$. Now let us take $q_{t}=\lambda q_{r}$ and $p_{t}=\lambda p_{r}$, then $u_{t r}=0$ and $u_{r+s, t}=u_{s t}$ and so $F_{t r}^{q}=0$ and $F_{r+s, t}^{q}=F_{s t}^{q}$. Thus eq. (A2) implies

$$
F_{r, s+t}=F_{r s},
$$

or in other words, the quantity $F\left(q_{r}, p_{r} ; q_{s}+\lambda q_{r}, p_{s}+\lambda p_{r}\right)$ is actually independent of $\lambda$. Choosing $\lambda=-p_{s} / p_{r}$ yields

$$
F_{r s}=F\left(q_{r}, p_{r} ; \frac{u_{r s}}{p_{r}}, 0\right) .
$$

Likewise, using an analogous argument or using antisymmetry, $F\left(q_{r}+\lambda q_{s}, p_{r}+\lambda p_{s} ; q_{s}, p_{s}\right)$ is also independent of $\lambda$, thus

$$
F_{r s}=F\left(0, p_{r} ; \frac{u_{r s}}{p_{r}}, 0\right) .
$$

This implies that $F_{r s}$ depends at most on $u_{r s}$ and $p_{r}$. A similar argument shows that $F_{r s}$ may depend at most on $u_{r s}$ and $q_{r}$, therefore it depends only on $u_{r s}$.

Everything can be repeated for the dependence of $F$ on the classical variables using that $F_{r s}^{c}=v_{r s}$ is an antisymmetric function of $v_{r s}=k_{r} x_{s}-x_{r} k_{s}$. Thus, $F_{r s}$ depends only on $u_{r s}$ and $v_{r s}$.

Next, let us prove eq. (33). First of all, note that the three variables $x^{u}=u_{s t}, y^{u}=u_{t r}$ and $z^{u}=u_{r s}$ are independent, i.e, the triple $\left(x^{u}, y^{u}, z^{u}\right)$ can take any value in $\mathrm{R}^{3}$, and similarly $x^{v}, y^{v}$ and $z^{v}$ in the classical sector. Second, the structure of eqs. (32) is two dimensional but can naturally be extended to any number of dimensions. Let us denote by $x^{i}, i=1, \ldots, n$ the corresponding variables, i.e., $F=F(x)$ (in our particular case, $n=2$ with $x^{1}=u$ and $x^{2}=v$ ). Then the equations take the form

$$
\begin{aligned}
& F(x)=-F(-x), \\
& F(x) F(y-z)+F(y) F(z-x)+F(z) F(x-y)=0, \\
& F_{i}(0)=1,
\end{aligned}
$$

where $F_{i}(x):=\partial_{i} F(x)$. The last relation comes from $\partial_{u} F(0,0)=\partial_{v} F(0,0)=1$ which follows from the postulates. (Actually, only $\partial_{i} F(0) \neq 0$ is essential for the present argument). It is clear from the formulas that if $f$ is a one dimensional solution of these equations,

$$
F(x)=f\left(x^{1}+\cdots+x^{n}\right)
$$

is also a solution of the $n$-dimensional problem. Let us show that this is, in fact, the general solution. Applying $\partial^{2} / \partial y^{i} \partial z^{j}$ at $y=z=0$ to the Jacobi identity in eq. (A6) yields

$$
0=-F(x) F_{i j}(0)+F_{i}(0) F_{j}(-x)-F_{j}(0) F_{i}(x),
$$

that is, $F_{i}(x)=F_{j}(x)$ for all $i, j$. This implies eq. (A7). (This can be seen in the two dimensional case by making a change of variables to $x^{1}+x^{2}$ and $x^{1}-x^{2}$, and by induction for the higher dimensional cases.) 
The form $F(x)=f\left(x^{1}+\cdots+x^{n}\right)$ is already incompatible with the axioms, since the classical sector requires $f(x)=x$ whereas the quantum sector requires $f(x)=2 \sin (x / 2)$. In order to find the general form of $f(x)$ it is sufficient take the first derivative on $z$ at $z=x$ and then the first derivative on $y$ at $y=0$ in the Jacobi identity for $f$. This yields

$$
0=f(x) f^{\prime \prime}(x)+f^{\prime 2}(0)-f^{\prime 2}(x),
$$

which together with $f(-x)=-f(x)$ and $f^{\prime}(0)=1$ yields $f(x)=\frac{1}{h} \sin (h x)$ or $f(x)=x$. 


\section{REFERENCES}

[1] J. Maddox, Nature 373 (1995) 469.

[2] L. Rosenfeld, Nucl. Phys. 40 (1963) 353.

[3] R.H. Brandenberger, Rev. Mod. Phys. 57 (1985) 1.

[4] W. Boucher and J. Traschen, Phys. Rev. D37 (1988) 3522.

[5] A. Anderson, Phys. Rev. Lett. 74 (1995) 621.

[6] L.L. Salcedo, hep-th/9410007.

[7] B.S. DeWitt, J. Math. Phys. 3 (1962) 619.

[8] I.V. Aleksandrov, Z. Naturforsch. Teil A36 (1981) 902.

[9] K.R.W. Jones, Phys. Rev. A50 (1994) 1062.

[10] L.L. Salcedo, Phys. Rev. A54 (1996) 3657.

[11] L. Diósi, Quantum Semiclass. Opt. 8 (1996) 309.

[12] O.V. Prezhdo and V.V. Kisil, Phys. Rev. A56 (1997) 162.

[13] L. Diosi and J.J. Halliwell, Phys. Rev. Lett. 81 (1998) 2846.

[14] L. Van Hove, Mem. Acad. Roy. Belg., 26 (1951).

[15] G. Parisi and Y. Wu, Sci. Sin. 24 (1981) 483.

[16] P.H. Damgaard and H. Huffel, Phys. Rept. 152 (1987) 227.

[17] M. Namiki, I. Ohba, K. Okano, Y. Yamanaka, A.K. Kapoor, H. Nakazato and S. Tanaka, Stochastic quantization, Lecture notes in physics, Springer-Verlag, (Berlin, Germany, 1992).

[18] J. Glimm and A. Jaffe, Quantum Physics, Springer-Verlag (New York, USA, 1987). 